\title{
Students' determination of differential area elements in upper-division physics
}

\author{
Benjamin P. Schermerhorn and John R. Thompson \\ Department of Physics and Astronomy and Maine Center for Research in STEM Education, \\ University of Maine, Orono, ME, 04469, USA
}

\begin{abstract}
Given the significance of understanding differential area vectors in multivariable coordinate systems to the learning of electricity and magnetism $(\mathrm{E} \& \mathrm{M})$, students in junior-level $\mathrm{E} \& \mathrm{M}$ were interviewed about $\mathrm{E} \& \mathrm{M}$ tasks involving integration over areas. In one task, students set up an integral for the magnetic flux through a square loop. A second task asked students to set up an integral to solve for the electric field from a circular sheet of charge. Analysis identified several treatments of the differential area: (1) a product of differential lengths, (2) a sum of differential lengths, (3) a product of a constant length with differential length in one direction, (4) a derivative of the expression for a given area, and (5) the full area.
\end{abstract}

\section{INTRODUCTION}

The application of vector calculus in upper-division electricity and magnetism $(\mathrm{E} \& \mathrm{M})$ involves coordination of many conceptual aspects, including reasoning about the physical symmetry of the problem and constructing differential elements within particular coordinate systems. The differential area element, $d A$, is one particular aspect important to calculations in E\&M. It appears in expressions for electric and magnetic flux, where it is treated as a vector quantity. When applying Coulomb's Law to a surface charge distribution, students solve for the electric field by accumulating the effects of infinitesimal charges expressed in terms of a scalar differential area.

Research on student understanding of mathematics in E\&M found general student difficulties with setting up calculations, interpreting the results of calculations, and accounting for underlying spatial situations [1]. Work has explored students' applications of Gauss's and Ampère's laws [1-6] or broadly addressed student understanding of integration and differentials [7-10], in one task identify students were unsure of the meaning of dA in an integral over a charge density [10]. Despite this, few studies have explored student understanding of differential line, area, or volume elements as they are constructed or determined in the non-Cartesian coordinate systems employed in E\&M [11-12]. Pepper and colleagues [1] reported how a group of students neglected to include necessary scaling factors when writing spherical differential areas, using $d A=d \theta d \phi$ rather than $d A=r^{2} \sin \theta d \theta d \phi$; another group used $d x d y d z$ as a length element in a three-dimensional line integral. These errors demonstrate a limitation in students' understanding of how to construct and apply differential elements.

Some problems (e.g., those invoking Gauss's law) involve making symmetry arguments that bypass the determination of $d A$ and allow for the input of the full area, potentially obscuring students' understanding in problems where $d A$ construction is necessitated. As part of a project to investigate how students build or make determinations about multivariable differential elements, we interviewed junior-level E\&M students and asked them to explain their choice of $d A$ for various E\&M scenarios. Tasks included bother scalar and vector dA to elicit students' evoked concept images [13] of the differential area element.

\section{STUDY DESIGN \& METHODS}

In order to examine how students determine differential area elements, we developed two tasks involving integration over a given area. Both tasks are adapted from standard problems in the widely used course text [14].

In the first task, students were given the expression for the magnitude of the magnetic field induced by a long straight current-carrying wire and asked the find the magnetic flux through a square loop (Fig. 1). The varying magnetic field requires an integral expression for flux, $\Phi_{B}=\iint \vec{B} \cdot d \vec{A}$. This leads students to consider the differential area as a vector quantity. Given the curling nature of the magnetic field, cylindrical coordinates are optimal, but Cartesian coordinates can be used if students rewrite the magnetic field with the appropriate variable.

In the second task, students were asked to construct an integral to solve for the electric field a distance $x$ from a circular sheet of constant charge density, $\sigma$ (Fig. 2). This involves using Coulomb's Law, $\vec{E}=k \int d q(\vec{r}-$ $\left.\vec{r}^{\prime}\right) /\left(\vec{r}-\vec{r}^{\prime}\right)^{3 / 2}$, from electrostatics, where $d q$ is a differential charge and $\left(\vec{r}-\vec{r}^{\prime}\right)$ is a displacement vector from the location of $d q$ to the electric field measurement. Since the charge is distributed over a circular sheet, $d q$ can

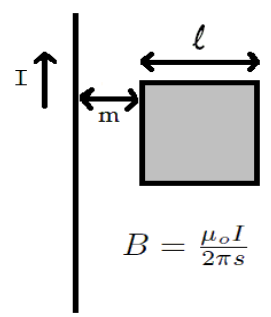

FIG 1. Image for task 1 depicting square loop (shaded) of side length $l$ at a distance $m$ from a current-carrying wire. 
be expressed as the product of the surface charge density and a differential area representing the charged surface.

These tasks were administered as parts of multi-task interviews to students in the second semester of junior-level E\&M at two universities. Two pair interviews (student designations $\mathrm{B} \& \mathrm{H}$ and $\mathrm{D} \& \mathrm{~V}$ ) were conducted at University A, followed by six other individual interviews $(\mathrm{J}, \mathrm{K}, \mathrm{L}, \mathrm{M}$, $\mathrm{N}$, and $\mathrm{O}$ ) with a different set of tasks the subsequent year. Interviews at University B involved two pairs and one individual student (P\&Q, R\&S, and T). Pair interviews were only given the flux task. Individual interviews featured both of the described tasks, separated by a line integral task. Pseudonyms are provided for students corresponding to their identifying letter (i.e., Jake for J).

As part of both interview questions, after completing the task students were asked to elaborate on their choices of differential areas in terms of how it was chosen or why it contained particular components. Interviews were videoed and later transcribed. Transcriptions and video data were analyzed to seek commonalities in students' treatment of differential areas, as well as related difficulties, using a concept images framework from mathematics education [13]. A student's concept image is a multifaceted and dynamic construct, including any ideas, processes and figures the student associates with a topic. A concept image for integration may contain area under the curve or Riemann sums [7], or be a specific rule such as that the indefinite integral of $n x^{n-1} d x$ is $x^{n}+C$, without the specific understanding of why that is the result. The particular aspect(s) called forth, referred to as the evoked concept image(s), depends on the task and context. Our analysis sought to identify evoked concept images of differential areas elicited during integration in E\&M tasks.

\section{RESULTS \& DISCUSSION}

From students' progression through the interviews, we identified several particular concept images of the differential area evoked across students' integral construction. Students commonly treated the differential area as constructed of differential lengths, which was largely productive. Due to attention or inattention to other ideas, the specific nature of the concept image ranged from a product of differential lengths to an incorrect sum of differential lengths to the product of a constant length with a differential in one direction. In other cases, students represented the differential area as the derivative of the expression for the given area, or as the full area itself. Ideas related to using the full area to construct $d A$ were a hindrance to students in the absence of high symmetry. These five processes for constructing the differential area encapsulate all interviewed students' choices for these two specific tasks. Additionally, several students' evoked concept images varied over the course of the interview task, reflecting a multifaceted concept image.

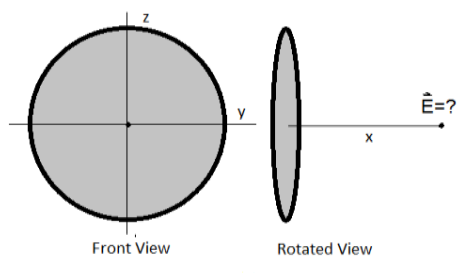

FIG 2. Image for task two of a charged sheet (shaded), with front and rotated view.

\section{A. Small portion of area constructed from differential lengths}

\section{Product of differential lengths}

Treatment of the differential area as a product of differential lengths was productive for students and most typically led to the correct expression. This entailed students recognizing a differential area on a particular surface as a product of two small changes in two given directions, respective to the needed area and the given coordinate system.

Students typically approached the first task with a Cartesian coordinate system, attending more to the square shape of the loop rather than the curling magnetic field:

Molly: Since it's a square, Cartesian coordinates would just be the easiest to integrate over it, so that would just be like a little bit, like the differential area is just a little bit in the $x$ and then a little bit in the $y$.

Thus the two differentials here were a combination of two of a $d x$ and $d y$, or $d y$ and $d z$, depending on how students placed their Cartesian axes. Three other students expressed similar reasoning with their choices of differentials, using either the idea of little changes in the necessary variables or referring to specific Cartesian axes.

This line of reasoning was very productive for the circular charged sheet task, where students more easily recognized the need for polar coordinates. Because the differential area in polar coordinates is not a simple square, students needed to include the necessary scaling factors.

Molly: ...to create a differential area on this circle we have we'd move a little bit $d s$ and then we'd move a little $d \varphi$, which is, well a little bit in the $\hat{\varphi}$ direction. Which is $s d \varphi$ because of the arc length formula.

Only two other students were able to correctly recognize the need for the radius in the expression for arc length. Kyle specifically wrote out the differential length for spherical coordinates, from which he'd chosen the two appropriate lengths, explaining $d A$ as "length times length" (Fig. 3). A fourth student recognized the need for two lengths but used the full radius of the circle for his arc length, which he treated as a constant during his integration. 


$$
\begin{aligned}
& d \vec{l}=d r \hat{i}+r d \theta \hat{\theta}+r \sin \theta \hat{\theta} \\
& d \vec{a}=d l_{1} \cdot d l_{2} \ldots \\
& d a=r d \theta d r
\end{aligned}
$$

FIG 3. Kyle's explanation for his choice of $d A$ in task 2, where he selects appropriate differential length elements from the generic length vector.

Thus while he demonstrated an understanding of how to construct a differential area, he was unable to arrive at the appropriate expression.

\section{Rectangle with constant height and differential width}

This categorization is specific to task 1, where the magnetic field from the wire only changed in one direction. In two interviews, students reasoned about the physical symmetry and implicitly integrated in the direction parallel to the wire, producing an $l$ in the equation.

Lenny: ...ldl in the $\hat{n}$ direction... $d l$ being the length to integrate the field over... that $l$ I'd assume to be this one $l$ right here, which would make the area, but I wouldn't feel like I'd have to integrate because the field is constant on that portion... If $[s]$ was the distance away, so that would be like $d s$ maybe.

In effect this method adds up the magnetic flux through rectangular strips of height $l$ and width $d s$. Students reasoning this way used the physical geometry to obtain the right solution but bypassed a choice of a coordinate system.

\section{Sum of differential lengths}

Jake expressed $d A$ as a sum of lengths rather than as a product for both tasks for reasons expressed in task 2:

Jake: Actually no, it will be $d r d \theta$ because it's a surface area so I'll need two dimensions that my $\mathrm{d} \theta$ is probably going to come in from my $d q$. Because I should have a differential area shouldn't I, and a differential area should be $d r d \theta$ [writes $d r+r d \theta]$.

Jake's difficulty relates to symbolic expression. He clearly states a need to include two dimensions for an area but represents this as a summation rather than a product. Similarly in task 1, Jake represents his differential area as $d s \hat{s}+s d z \hat{z}$, using an incorrect differential length.

\section{B. Derivative of the area expression}

Students attempted to functionalize the given area and take a derivative to gain an expression for the differential area across three interviews. This is consistent with a conceptual metaphor of the derivative as a machine [8]: students interpret the $d$ in $d A$ as a need to differentiate the function represented by the second variable. For Jake and Tyler, the ensuing difficulty was what to take their derivative with respect to. Both decide to integrate with respect to $r$ (Fig. 4), which neglected the integrand's dependence on $\theta$. This caused Jake to switch back to his sum of differential lengths concept image.

A pair of students employed this idea for task 1:

Percy: ... you still need... $d$ something. I mean, what is your area? The area equals $l^{2}$ so da equals... $2 l d l . .$. What we would do is say: "Oh look at this, what I have is integral of some $d A$. Well, what is the area of this? Oh, that's $l^{2}$,.. We would just recognize the fact that it's an integral of... an area element, so we take the area of the object and we'd do it easy.

Here, Percy reasons about the differential area represented in their flux equation as just the derivative of the area in an attempt to justify his final answer as just the multiplication of the magnetic field with the area of the square. Neither student attends to the fact that the magnetic field is changing in one direction or would need to be constant to bypass the use of $d A$. This particular reasoning speaks more to the treatment of $d A$ as something that simply integrates to an area rather than a geometrical object accounting for integration in two different coordinate directions.

\section{The area of the region itself}

A third overall approach was to insert a functional form of the area for the whole region as $d A$. This was often the result of inattention to differentials and/or students' perceived need to plug in the area.

Bart: The $d A$ is the area of the square...you want just the square loop. I mean, there is flux everywhere but you want just the square loop. This is $B$ [gestures to summing of fields at each edge of the loop] and $[d A]$ is $l^{2}$.

Throughout the interview Bart was persistent about plugging in the area, much in the way Percy was above. However for Bart, the area being $l^{2}$ was subsumed into the integral, which then resulted in a multiplication of his (incorrect) magnetic field and the full area. This was not something on which these students sought consistency.

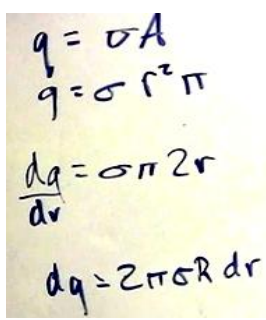

FIG 4. Jake's first attempt at solution on task 2, starting with an expression for area and deriving a differential form. 
Nate applied this reasoning to both tasks, replacing $d A$ with the perceived area of the given space. Nate included these differentials in his integrals in an attempt to identify what quantities needed to be integrated over (Fig. 5). Nate's explanation later in the interview of task 1 illustrated an understanding of the physical nature of $d A$ as a "little chunk of area," an idea that Nate failed to connect to his earlier representation or to his addition of differentials. Nate's treatment of the differentials $d x$ and $d y$ is consistent with the differential as a nonphysical quantity, or just a variable of integration [8]. These conceptions of both $d A$ and differentials persisted into task 2 , where Nate described the area of a circle as $\cos ^{2} \theta$, which would be multiplied by $\sigma$ to express the differential charge $d q$.

As depicted, students attempting to express the differential area with an equation for the area of the full region have additional trouble with other parts of the tasks.

\section{CONCLUSIONS}

Analysis of student interviews covering typical E\&M tasks allowed us to identify several concept images of the differential area elicited from students. As part of a larger integration task, the $d A$ was commonly treated as a small portion of area constructed from differential lengths, as the derivative of the given area, or as the given area itself. Notably, the particular solution method employed was independent of coordinate system, suggesting students' methods for determining differential areas are detached from students' perceived coordinate system.

The most productive instantiation of students' concept images was to express the differential area in terms of a product of differential lengths. This was especially productive for students working in polar coordinates, where they were not able to use aspects of the physical system to bypass defining a differential area. Other students possessed correct ideas pertaining to differential area but either had difficulty with the correct expression of individual differential lengths or displayed confusion with the overall symbolic template of the expression (e.g., added lengths).

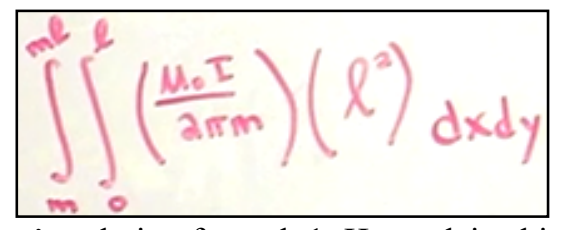

FIG 5. Nate's solution for task 1. He explains his choice of $d A$ as $l^{2}$ and the inclusion of $d x d y$ due to the need to integrate over the given boundaries.

Students incorrectly expressing differential areas most commonly focused on the final area of the given region, whether attempting to take a derivative to account for the need to integrate or forcibly inserting a function for the full area into the integral. Emphasis on plugging in the area is most likely an artifact of generalizing common textbook problems that are highly symmetric, such as Gauss's law, where they can "do it easy" and neglect the dot product and vector nature of the $d A$, and simply express the integral as a product of the field and the area of a Gaussian surface. While in very specific cases inserting a given area or the derivative of said area may produce a correct result, such as Jake's derivative of area response for task 2, where symmetry negates the need to integrate over $\theta$, these methods are not as universal as students perceive them to be. Students' use of area in this way is another example of overuse of symmetry arguments in problems where symmetry is not present [1-3].

Results suggest that an explicit instructional focus on the construction of differential areas as the product of differential lengths in specific coordinate systems, even in high-symmetry situations, may help dissuade students' overemphasis on a "plugging in the area" approach.

\section{ACKNOWLEDGEMENTS}

The authors would like to acknowledge Michael Loverude and Mikayla Mays for their organizational assistance. This material is based upon work supported by NSF grant PHY-1405726.
[1] R. Pepper, S. Chasteen, S. Pollock, and K. Perkins, Phys. Rev. ST - Phys. Educ. Res., 8, 010111 (2012).

[2] A. Traxler, K. Black, and J. R. Thompson, 2006 Phys. Educ. Res. Conf. AIP CP, 883, 173-176 (2006).

[3] C. Singh, Am. J. Phys., 74, 923-936, (2006).

[4] C. Manogue, K. Browne, T. Dray, and B. Edwards, Am. J. Phys., 74, 344-350 (2006).

[5] C. Wallace and S. Chasteen, Phys. Rev. ST - Phys. Educ. Res., 6, 020115 (2010).

[6] J. Guisasola, J. Almudí, J. Salinas, K. Zuza, and M. Ceberio, Eur. J. Phys., 29, 1005-1016 (2008).

[7] L. Doughty, E. McLoughlin, and P. van Kampen, Am. J. Phys., 82, 1093-1103 (2014).

[8] D. Hu and N. S. Rebello, Phys. Rev. ST - Phys. Educ. Res., 9, 020108 (2013).
[9] D. Meredith and K. Marrongelle, Am. J. Phys., 76, 570-578 (2008).

[10] D. Nguyen and N. S. Rebello, Phys. Rev. ST - Phys. Educ. Res., 7, 010113 (2011).

[11] B. Schermerhorn and J. R. Thompson, Proc. $19^{\text {th }}$ Ann. Conf. on Res. in Undergraduate Math. Educ. MAA, (2016).

[12] B. Schermerhorn and J. R. Thompson, 2016 Phys. Educ. Res. Conf. AIP CP, 312-315 (2016).

[13] D. Tall and S. Vinner, Educ. Stud. Math., 12, 151169 (1981).

[14] D. Griffiths, Introduction to Electrodynamics (4th edition) (Pearson, USA, 2013). 